\title{
TAKE A CLOSER LOOK AT TRIALS
}

\section{Global Consortium Study of Neurological Dysfunction in COVID-19 (GCS-NeuroCOVID): Study Design and Rationale}

Jennifer Frontera ${ }^{1 \dagger}$, Shraddha Mainalii ${ }^{2 \dagger}$, Ericka L. Fink ${ }^{3}$, Courtney L. Robertson ${ }^{4}$, Michelle Schober ${ }^{5}$, Wendy Ziai ${ }^{6}$, David Menon?7, Patrick M. Kochanek ${ }^{8}$, Jose I. Suarez ${ }^{6}$, Raimund Helbok ${ }^{9}$, Molly McNett ${ }^{10^{*+}}$ and Sherry H.-Y. Chou ${ }^{11+}$ on behalf of the GCS-NeuroCOVID Study

(c) 2020 Springer Science+Business Media, LLC, part of Springer Nature and Neurocritical Care Society

\begin{abstract}
Background: As the COVID-19 pandemic developed, reports of neurological dysfunctions spanning the central and peripheral nervous systems have emerged. The spectrum of acute neurological dysfunctions may implicate direct viral invasion, para-infectious complications, neurological manifestations of systemic diseases, or co-incident neurological dysfunction in the context of high SARS-CoV-2 prevalence. A rapid and pragmatic approach to understanding the prevalence, phenotypes, pathophysiology and prognostic implications of COVID-19 neurological syndromes is urgently needed.
\end{abstract}

Methods: The Global Consortium to Study Neurological dysfunction in COVID-19 (GCS-NeuroCOVID), endorsed by the Neurocritical Care Society (NCS), was rapidly established to address this need in a tiered approach. Tier-1 consists of focused, pragmatic, low-cost, observational common data element (CDE) collection, which can be launched immediately at many sites in the first phase of this pandemic and is designed for expedited ethical board review with waiver-of-consent. Tier 2 consists of prospective functional and cognitive outcomes assessments with more detailed clinical, laboratory and radiographic data collection that would require informed consent. Tier 3 overlays Tiers 1 and 2 with experimental molecular, electrophysiology, pathology and imaging studies with longitudinal outcomes assessment and would require centers with specific resources. A multicenter pediatrics core has developed and launched a parallel study focusing on patients ages $<18$ years.

Study sites are eligible for participation if they provide clinical care to COVID-19 patients and are able to conduct patient-oriented research under approval of an internal or global ethics committee. Hospitalized pediatric and adult patients with SARS-CoV-2 and with acute neurological signs or symptoms are eligible to participate. The primary study outcome is the overall prevalence of neurological complications among hospitalized COVID-19 patients, which will be calculated by pooled estimates of each neurological finding divided by the average census of COVID-19 positive patients over the study period. Secondary outcomes include: in-hospital, 30 and 90-day morality, discharge modified Rankin score, ventilator-free survival, ventilator days, discharge disposition, and hospital length of stay.

\footnotetext{
*Correspondence: mcnett.21@osu.edu

†Jennifer Frontera and Shraddha Mainali: first authors contributed equally.

${ }^{\dagger}$ Molly McNett and Sherry H.-Y. Chou: last authors contributed equally.

${ }^{10}$ College of Nursing, The Ohio State University, 760 Kinnear Rd,

Columbus, $\mathrm{OH}$ 43212, USA

Full list of author information is available at the end of the article
}

\section{实 Springer}


Results: In a one-month period (3/27/20-4/27/20) the GCS-NeuroCOVID consortium was able to recruit 71 adult study sites, representing 17 countries and 5 continents and 34 pediatrics study sites.

Conclusions: This is one of the first large-scale global research collaboratives urgently assembled to evaluate acute neurological events in the context of a pandemic. The innovative and pragmatic tiered study approach has allowed for rapid recruitment and activation of numerous sites across the world-an approach essential to capture real-time critical neurological data to inform treatment strategies in this pandemic crisis.

Keywords: COVID-19, SARS-CoV-2, Neurological manifestations, Neurological symptoms, Coronavirus

\section{Introduction}

As the COVID-19 pandemic evolves worldwide, reports of a spectrum of mild to severe neurological syndromes among patients infected with SARS-CoV-2 are emerging, including headache, anosmia, ageusia, seizures, coma, encephalitis, Guillain-Barre syndrome, and acute cerebrovascular events including ischemic stroke, intracerebral hemorrhage, and cerebral venous sinus thromboses [1-14]. Early reports from China suggested that neurological symptoms may be found in up to $36 \%$ of COVID19-positive patients, with higher prevalence among more severe cases [6]. Helms et al. [15] reported that $84 \%$ $(49 / 58)$ of patients with severe SARS-CoV-2 infection and acute respiratory distress syndrome had neurological symptoms including encephalopathy, agitation and confusion, and corticospinal tract signs. However, very little is known of the etiology of these reported neurological findings in the context of SARS-CoV-2 infection. Potential possibilities include direct viral invasion, para-infectious mechanisms, secondary neurological manifestations of systemic organ failure, or coincident neurological dysfunction in the context of high SARS$\mathrm{CoV}-2$ population prevalence [16].

We established the Global Consortium to Study Neurological dysfunction in COVID-19 patients (GCSNeuroCOVID) and promptly launched a tiered research program with an early, pragmatic, and nimble design to enable successful implementation during a global pandemic crisis when healthcare systems are stressed. The over-arching goals of this consortium are: (1) to characterize and estimate the prevalence of neurological syndromes in hospitalized patients with SARS-CoV-2 infection; (2) to identify predictors of neurological dysfunction among COVID-19 patients; (3) to determine the impact of neurological dysfunctions on functional and cognitive outcomes of COVID-19 survivors; and (4) to utilize routine and experimental laboratory, electrophysiologic, and radiographic biomarkers to decipher potential mechanisms and predict outcome of neurological injuries in COVID-19 infection.

\section{Methods \\ Study Rationale \\ Biological Plausibility for Neurotropism}

The SARS-CoV-2 virus shares significant structural homology with SARS-CoV, which causes SARS and is known to have a variety of neurological manifestations [17-20] and a predilection for neurons [21-23]. To enter cells, coronaviruses must have their viral spike (S) protein bind to a cell surface receptor as well as S-protein priming by host cell proteases. Both SARS-CoV and SARS-CoV-2 use the cell surface receptor angiotensinconverting enzyme 2 receptor (ACE2R) for cell entry. Neurons and glial cells express ACE2R [24]. Meanwhile, oligodendrocyte precursor cells and astrocytes express the serine protease TMPRSS2 required for SARS-CoV-2 S-protein priming $[24,25]$. In one postmortem tissue study, Paniz-Mondolfi et al. [26] described viral presence in frontal lobe neural and capillary endothelial cells from a patient infected with SARS-CoV-2. Taken together, these facts suggest biological plausibility that SARSCoV-2 may enter the CNS.

Emerging reports of prominent anosmia and dysgeusia symptoms among COVID-19 patients led many to postulate that SARS-CoV-2 may enter the brain from the nasopharynx via retrograde viral migration along the olfactory bulb or directly invade the CNS through the cribriform plate [27, 28]. However, evidence of SARSCoV-2 in human cerebrospinal fluid (CSF) or brain tissue specimens is lacking $[2,15,29]$. One case report from Japan identified SARS-CoV-2 RNA in patient's CSF obtained 9 days after initial COVID-19 symptom onset [14]. However, nasopharyngeal swabs obtained at the same time were negative, raising the possibility of either a contaminated specimen or viral neurotropism that may exist independent of systemic involvement [14].

Similar to adults, children experience a spectrum of neurological manifestations in response to viral infection and post-infectious immune dysfunction. Recent evidence highlights acute and long-term neurological manifestations due to other viral illnesses including Guillain-Barre syndrome and neonatal microcephaly with Zika virus, encephalitis [30] with H1N1 influenza [31], and acute flaccid paralysis with enterovirus-68 
[32]. However, COVID-19 reports in children published thus far lack detailed information on the frequency and outcomes of neurological findings on presentation and through hospital admission [33].

\section{Establishing Prevalence Data}

Reports of potential neurological dysfunctions observed in COVID-19 patients have emerged at an increasing pace. Initially, many such reports were from verbal communications between colleagues or presented online in preprint form prior to scientific peer review. Of the peer-reviewed, published case reports/case series to date, most contain less objective/diagnostics data compared to pre-COVID-19 era, likely due to limitations related to COVID-19 containment and healthcare resource limitations in a pandemic. Reported SARS-CoV-2-related neurological syndromes include ischemic [34] and hemorrhagic strokes [3], Guillain-Barre syndrome [4], a case of necrotizing encephalopathy [2], and a case of atypical presentation of COVID-19 in infants [35]. Nearly all reported cases of neurological dysfunction were in hospitalized patients. None have reported the underlying COVID-19 prevalence rate in the hospitalized patients at the times of these cases. Identifying neurological phenotypes of SARS-CoV-2 may identify an important fraction of COVID-19 patients who have non-classical presentation with neurological symptoms, which may, in turn, change screening strategies. Infants, children, and adolescents may have very different SARS-CoV-2 presentations than adults. Understanding neuro-phenotypes is particularly important in the pediatric population, for which COVID-19 data have generally been sparse. Identifying post-infectious neurological events that can occur subsequent to the diagnosis and may impact long-term outcomes (e.g., cognitive, emotional, physical health) is equally critical.

\section{Understanding the Impact on Outcome}

The spectrum of neurological dysfunction observed in the context of SARS-CoV-2 may have variable effect on patient outcome. This study will identify in-hospital and delayed functional and cognitive outcomes that may assist in prognostication and goals of care discussions among COVID-19 patients. Additionally, variables addressing code status and withdrawal of life-sustaining therapy are collected, which will allow for modification of mortality estimates based on code status.

\section{Insight into Treatment Strategies}

Exploratory analysis of this planned observational study may also provide information on potential impact of empiric COVID-19 therapeutics on emerging neurological syndromes. The effect of medication(s) given empirically and in controlled trials for the treatment of COVID-19 infection or para-infection on neurological symptoms is unknown. Ideally, once we characterize the main phenotypes of neurological syndromes seen in COVID-19, the global community can develop treatment guidelines or systematic approaches to empiric therapies targeting neurological dysfunction. Similarly, in both the adult and pediatric populations, we anticipate that knowledge gained from this study regarding neurological manifestations will bolster personalized treatment and rehabilitation strategies to optimize outcomes and inform future interventional studies.

\section{Patient Population}

Table 1 summarizes inclusion and exclusion criteria for both adult and pediatric studies. The adult study will recruit COVID-19 patients exhibiting acute neurological signs or symptoms. For the adult study, patients with preexisting, severe, baseline neurological dysfunctions such as baseline coma/vegetative state, quadriparesis, or refractory seizure disorder with frequent breakthrough despite anticonvulsant therapy are excluded because discerning new from existing neurological dysfunction

Table 1 Tier 1 study inclusion and exclusion criteria

\begin{tabular}{|c|c|}
\hline Inclusion criteria & Exclusion criteria \\
\hline \multicolumn{2}{|l|}{ Adult study (age $\geq 18$ years) } \\
\hline $\begin{array}{l}\text { Admitted to acute care hospital with confirmed COVID-19 infection or } \\
\text { COVID-19 suspect patient under investigation (PUI) } \\
\text { Patient exhibiting acute neurological symptom(s)/dysfunction(s) }\end{array}$ & $\begin{array}{l}\text { Outpatient status } \\
\text { Lack of specific new neurological findings } \\
\text { Preexisting, severe, baseline neurological dysfunction including baseline } \\
\text { coma/vegetative state, baseline quadriparesis, baseline refractory } \\
\text { seizure disorder with frequent breakthrough seizures despite anticon- } \\
\text { vulsant use }\end{array}$ \\
\hline \multicolumn{2}{|l|}{ Pediatric study (age < 18 years) } \\
\hline $\begin{array}{l}\text { Children age }<18 \text { admitted to acute care hospital with confirmed or pre- } \\
\text { sumed COVID-19 }\end{array}$ & None \\
\hline
\end{tabular}


would be difficult. For the pediatric study, children under the age of 18 years who are hospitalized with confirmed or presumed COVID-19 are eligible; there are no exclusion criteria.

We will recruit patients who are documented to have COVID-19 (either through detection of SARS-CoV-2 in a relevant biosample, or through appropriate serology) as well as COVID-19 Patients Under Investigation (PUI), in whom clinical suspicion for SARS-CoV-2 infection is high. Inclusion of the latter category is justified for several reasons. First, testing availability differs broadly by location and center. Centers may have limited testing or repeat-testing capabilities for COVID-19 in patients with highly suspicious clinical syndromes. Second, laboratory COVID-19 test turnaround times for diagnosis vary significantly by location and center. At sites that require over $24 \mathrm{~h}$ for results to be available, patients could first enter the study by qualifying as COVID-19 PUI and later have confirmatory results. Third, COVID-19 test false negative rates vary by center. Patients with negative COVID-19 test results but highly suspicious clinical syndrome would remain as COVID-19 PUI and should be included. Finally, some patients who have undergone testing (and hence qualify as COVID-19 PUI) may die or leave the hospital before COVID-19 results are available. We believe that inclusion of COVID PUI subjects would therefore increase the sensitivity for case ascertainment. We will address the potential reduced specificity inherent in this approach by comparing primary and secondary outcomes in laboratory-confirmed COVID-19 patients to PUI patients.

\section{Study Design}

GCS-NeuroCOVID is a prospective, international, multicentered, tiered study, designed to maximize site involvement at minimal cost, while preserving scalability. The pragmatic design has three tiers of study involvement ranging from IRB-exempt basic clinical data element collection (CDE) with waiver of consent all the way to advanced translational laboratory, electrophysiologic, and imaging studies to probe into pathophysiology (Table 2). The Neurological Manifestations of COVID-19 pediatrics study is a multinational, observational study organized similarly to the adult study with three planned tiers.

\section{Tier 1}

Tier 1 is a focused, prospective observational study, pragmatically designed and accounting for the many logistical limitations in patient-centered research during the current COVID-19 pandemic. This is a prevalence study specifically designed to qualify for waiver of consent and expedited internal review board (IRB)/ ethics board review. Given hospitals and intensive care units (ICUs) are locked down and in-person research activity suspended in many locations around the globe, Tier 1 is specifically designed to avoid any in-person patient contact or any need to enter the hospital for data collection, thus minimizing staff exposure risk and preserving personal protective equipment (PPE) for necessary clinical use. Another important pragmatic feature for Tier 1 study design is the separation of a very basic core set of data elements that need to be collected in real time, plus a set of "supplemental-highly recommended" data that, if necessary, can be obtained retrospectively from patient records with relative accuracy. Examples of core data elements include dates of neurological symptom onset and elements of the neurological assessment. Examples of "supplemental-highly recommended" data elements include basic laboratory values such as leukocyte counts. Overall, Tier 1 study is specifically designed to be very focused with low data collection burden such that all centers, regardless of level of research support and overall patient volume, may successfully implement this study with support from the central coordinating center.

The pediatric Tier 1 is designed to capture detailed information due to the smaller number of children who meet these criteria. There are two aims for Tier 1: (1) quantify neurological symptoms and diagnoses during hospitalization; (2) examine associations between empiric COVID-19-directed therapies and patient characteristics with outcomes.

\section{Tier 2}

Overlaying Tier 1, Tier 2 study will capture more detailed data on clinical examination findings, routine clinical laboratory, electrophysiology, imaging, and microbiological data that were not captured in Tier 1 due to severe research logistic limitations in the first phase of this pandemic. In addition, Tier 2 study will assess longterm mortality and functional outcomes beyond acute care hospital discharge. This study phase would require implementation of post-discharge longitudinal outcome evaluations via telecommunication tools and will include neurocognitive and quality-of-life assessments in addition to global outcome. The Tier 2 study would require study subject contact (by telecommunication to observe social distancing requirements) and would require informed consent from participants. By design, Tier 2 study will require more research personnel resources compared to Tier 1 and will therefore have fewer study sites and likely a smaller overall sample size, but more granular clinical data plus longitudinal outcome assessments. Since both Tiers 1 and 2 are observational studies, 


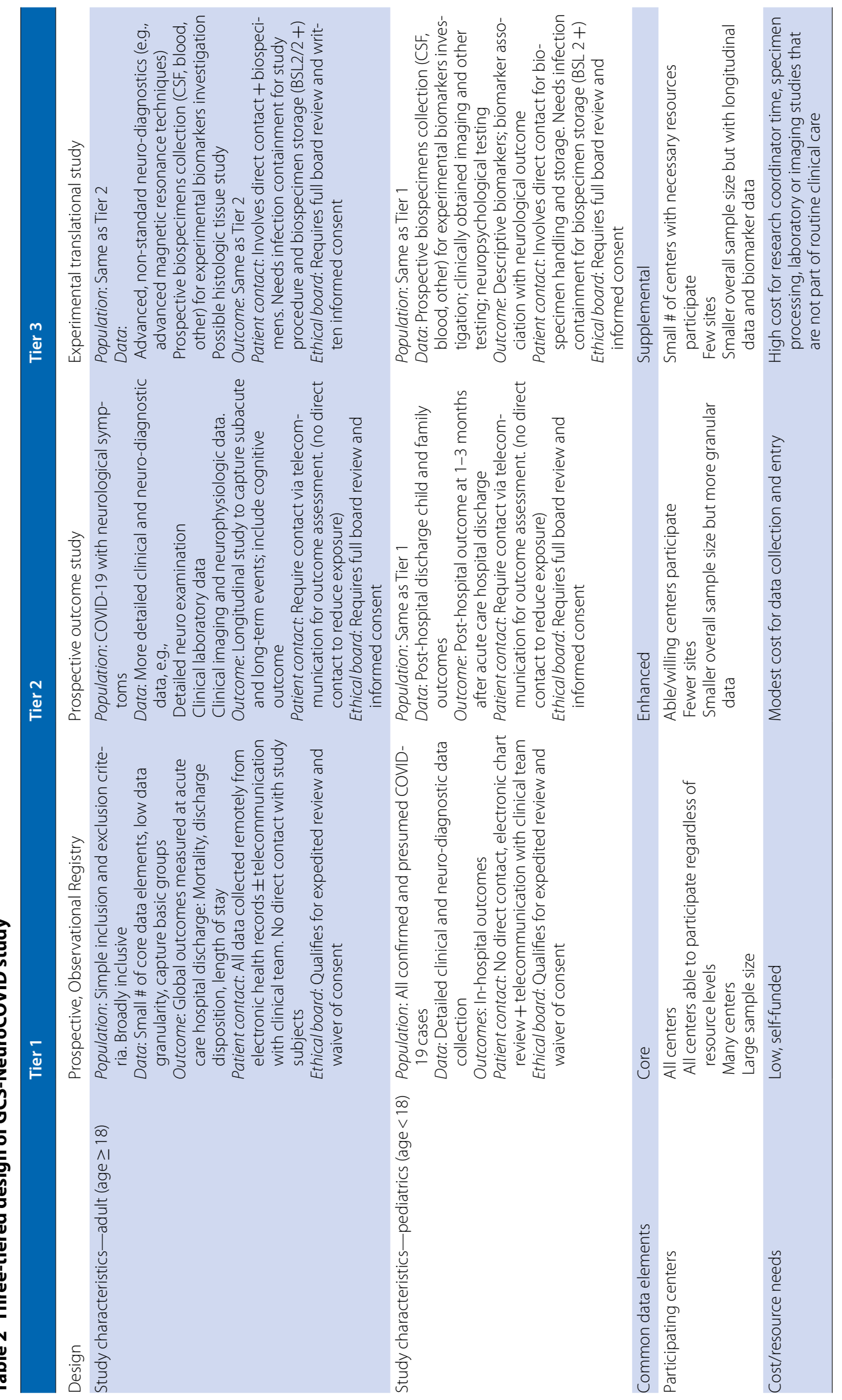


patients enrolled in these components of GCS-NeuroCOVID are also eligible to participate in interventional controlled clinical trials. This may allow us to explore potential effects of these investigational therapeutics on the prevalence and outcome of neurological dysfunctions in COVID-19.

The aim for Tier 2 in the pediatric study is to analyze child and family functional health and health-related quality-of-life outcomes between 1 and 3 months posthospital discharge by the presence or absence of any neurological symptoms.

\section{Tier 3}

Tier 3 is constructed as an advanced translational study that involves experimental protocols beyond routine clinical care. In this tier, advanced experimental neuroimaging, neurophysiologic, and molecular diagnostic techniques may be used to probe the physiology and pathophysiology of neurological dysfunctions and injuries in COVID-19. A centerpiece of Tier 3 is the prospective collection and banking of biospecimens such as blood and cerebrospinal fluid (CSF) during acute as well as subacute and chronic phases of disease to facilitate future advanced biomarker analysis. Tier 3 studies would require facilities with existing resources and expertise in advanced experimental imaging protocols and resources and facilities to prospectively bank biospecimens with the required level of security and biocontainment. Given these requirements, it is expected that only select sites with these resources can participate in Tier 3.

The pediatric Tier 3, similar to the adult study, focuses on creation of a biobank of prospectively collected CSF and blood specimens obtained during acute, subacute, and chronic phases of disease to facilitate future mechanistic and diagnostic analyses.

\section{Common Data Elements}

An important consideration of this international collaborative consortium was to develop global consensus around CDEs for neurological dysfunction in COVID19 to promote data harmonization and enable future merger of large datasets. Development and adaptation of CDEs have facilitated significant advancement and breakthrough in many clinical areas of investigation and is strongly endorsed by the United States National Institute of Health [36]. The National Institute of Neurological Disease and Stroke (NINDS) has developed and catalogued consensus CDEs across many important neurological conditions including stroke, subarachnoid hemorrhage, and traumatic brain injury [37-39]. CDE development for COVID-19 neurological dysfunction will follow the guidelines set forth by the NINDS CDE project [36, 37, 40-43] [44]. Tier 1 CDE includes patient sex, age, race/ethnicity, presence of comorbidities, neurological symptoms and syndromes, admission laboratory values, radiographic findings, pharmacological treatments and hospital outcomes including death, discharge disposition, and length of stay. Tier 2 and $3 \mathrm{CDE}$ are actively being developed.

\section{Outcome Assessments}

The primary outcome in Tier 1 is the prevalence of new clinical neurological syndromes in SARS-CoV-2 patients including: new onset headache, anosmia/ageusia, clinical seizures/status epilepticus, strokes (ischemic and hemorrhagic), meningitis/encephalitis, hypoxic/ischemic injury, acute encephalopathy, coma, myelopathy, neuropathy, and dysautonomia/sympathetic storming. Standard definitions are provided in the data dictionary to assist uniform adjudication of these clinical syndromes. Secondary outcomes include in-hospital, 30- and 90-day morality. Additional exploratory secondary outcomes include global functional outcome at acute care hospital discharge measured by modified Rankin score, ventilatorfree survival, discharge disposition, ventilator days, and hospital length of stay. The general design and outcome measures for Tiers 2 and 3 are under development and summarized in Table 1.

\section{Consortium Structure and Data Storage Coordination}

To expedite site initiation and data acquisition, case report forms and data dictionaries were distributed to local study sites allowing for immediate data entry. Similarly, IRB study templates were distributed to facilitate site readiness. Given the urgency of capturing data during SARS-CoV-2 patient surges, a parallel process was adopted whereby each site obtained single-center local IRB approval, while a data use agreement was developed by the data coordinating centers for future data consolidation. Given the global nature of this consortium, data storage and sharing are required to comply with all local and regional regulations (e.g., the General Data Protection Regulation).

Access to consortium data will be governed by a datasharing plan that makes the unique resources from this network available to the scientific community, while ensuring that the rights and privacy of human subjects who participate in research are protected at all times. We will do so by establishing a scientific committee comprised of international adult and pediatric subspecialist members from centers who have contributed data. All requests for consortium data will be submitted for review and adjudication through this scientific committee. Once approved by the scientific committee, data will be made available to requesting investigators after establishing a 
data-sharing agreement. De-identified data will be made publicly available upon the completion of the project and publication of primary manuscripts from this work.

\section{Site Registration}

Individual institutions/hospitals are invited to register for participation at https://www.neurocriticalcare.org/ resources/covid19. Each site is requested to identify a single-site principal investigator (PI) and one secondary contact person. Case report forms, data dictionaries, and IRB templates will be provided following successful site registration.

\section{Study Timeline}

Figure 1 demonstrates the GCS-NeuroCOVID study development in relationship to milestones of the unfolding SARS-CoV-2 pandemic. Tier 1 of GCS-NeuroCoV is designed to collect data for a 3-month interval. In 1 month from $3 / 27 / 20$ to $4 / 27 / 20$, over 70 international sites have registered for participation and several are currently collecting data. Collation of multicenter adult study data is projected to begin by August 2020. Analysis of Tier 1 data is projected to be completed by September 2020 with planned manuscript submission by October 2020. The pediatric study is planned to continue enrollment through December 2021 due to lower prevalence rates.

\section{Statistical Analyses}

The overall prevalence of neurological complications among hospitalized COVID-19 patients will be calculated by pooled estimates of neurological findings divided by total COVID-19-positive patients over the study period. Daily prevalence rates will be calculated by the number of COVID-19-positive or COVID-19 PUI patients with neurological dysfunctions divided by the total number of hospitalized COVID-19-positive or PUI patients on day of study enrollment. Descriptive statistics and frequency analysis will be used to describe demographic, clinical, and radiographic characteristics of the study sample and overall prevalence of confounding factors. Bivariate analysis including chi-square statistics for nominal and ordinal level variables and $t$ test or comparable nonparametric analysis for continuous-level variables will be performed to explore relationships between patient-level characteristics and study outcomes. Regression modeling will be performed to identify the impact of individual neurological complications on patient mortality, discharge disposition, length of stay, and functional and cognitive outcomes.

\section{Discussion}

This study represents the one of first large global efforts, to our knowledge, to establish a rapid, pragmatic paradigm to evaluate acute neurological events during a pandemic. We have demonstrated that expedited study design, deployment, and data collection are possible on

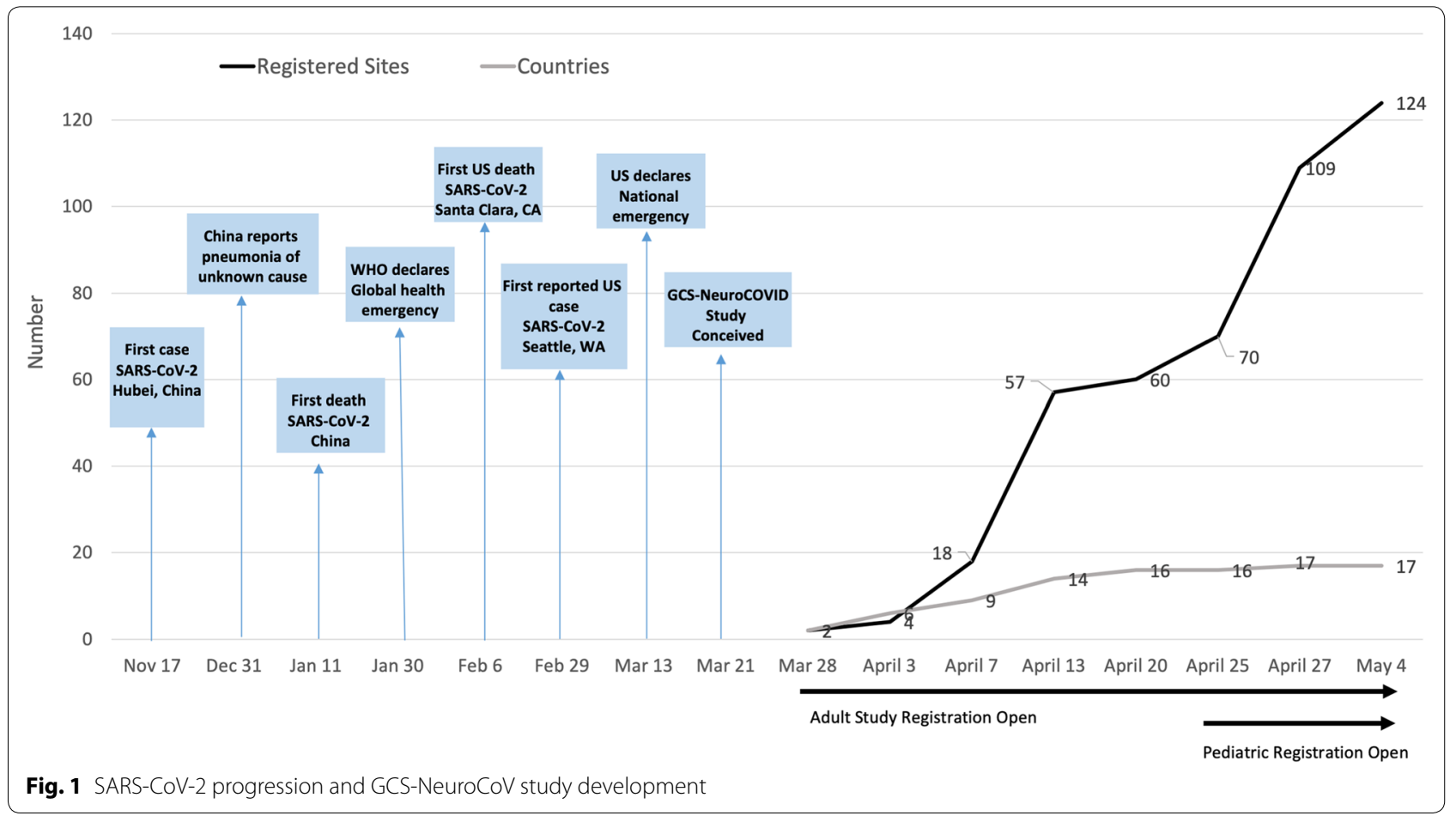


an international scale within a 1-month time frame and in the context of a global pandemic. This study may prove useful as a template for the development of other lowcost, global research initiatives.

Designing and implementing a large, international, collaborative study to investigate an emerging disease represents uncharted territory for most scientific investigators and research organizations. The severity of this pandemic and the exponential speed at which new information has emerged has demanded that we utilize innovative strategies, parallel processing, and a nimble and adaptive approach to study design. Strengths of this study include: (1) the tiered design, which allows for participation of a large number of sites internationally with minimal resource deployment, while maintaining scalability; (2) development of CDEs to harmonize data collection across sites; and (3) leveraging of existing NCS international partnerships for site recruitment. As we implement each tier, new and emergent information can be incorporated into design and development of next tier studies in real time. Furthermore, Tiers 2 and 3 will have a modular design, where data elements for each investigational or diagnostic technique (e.g., laboratory biomarkers, electrophysiology, or neuroimaging) may be developed and implemented in parallel, providing centers with the flexibility to implement select modules that are best suited to each institution's resources and patient population.

There were several critical considerations that the consortium sought to address while developing this study. First, our priority is to estimate the scope and prevalence of neurological sequelae of SARS-CoV-2. Though several case series have been published, there are no good estimates of the incidence or prevalence of neurological dysfunction, nor are there data evaluating the impact of neurological complications on outcome and long-term disability. While the lack of extensive testing makes it difficult to estimate the number of COVID-19 infections in the community, restricting the study population to hospitalized patients provides a reasonable denominator to begin to determine the prevalence of neurological dysfunction.

Second, a cautious evaluation of causal inference will be necessary when interpreting the data. For example, if both a virus and neurological syndrome are widely prevalent in a given population, the pathophysiologic link between entities becomes less robust. By the multicenter nature of this study, community prevalence of SARS-CoV-2 will vary across sites. Acute neurological abnormalities may also arise as secondary injuries due to multi-system organ dysfunction associated with SARSCoV-2 infection. For example, acute encephalopathic syndromes may be due to SARS-CoV-2-related renal failure, delirium may be due to prolonged intubation and sedation, stroke may be related to SARS-CoV-2-induced myocarditis, and myopathy and/or neuropathy may result from prolonged critical illness, neuromuscular blockade, or steroid use. Following Hill Criteria [45], if we are able to establish temporal relationships with infection preceding neurological symptoms, biological plausibility (e.g., biomarkers of a SARS-CoV-2-induced hypercoagulable state preceding an ischemic stroke), and consistent and strong relationships between SARS-CoV-2 infections and specific neurological syndromes, we will be more confident that we are observing a causal relationship. As a first step, we will characterize the phenotypes of neurological dysfunctions seen in COVID-19 based on rigorous clinical observations. Following this, additional laboratory, electrophysiologic, radiographic, and even histologic/tissue investigations can be used to investigate underlying pathophysiologic processes.

Third, while our study is designed to capture acute and early post-infectious neurological manifestations of COVID-19, there may be late cognitive and neurological syndromes that develop outside of our initial study time frame. The often-cited connection between encephalitis lethargica and the 1918 influenza pandemic exemplifies the possibility of a post-hospital discharge neurological sequelae, potentially driven by autoimmunity [46-48]. A prudent approach may include patient education, longitudinal follow-up care, and acute-late biospecimen analysis.

\section{Conclusions}

The Global Consortium to Study Neurological dysfunction in COVID-19 patients (GCS-NeuroCOVID) is one of the first large-scale, global research collaboratives urgently assembled to evaluate acute neurological events in the context of a pandemic. The innovative and pragmatic study approach has allowed for rapid recruitment and activation of over 70 sites across the world-an approach essential to capture real-time critical neurological data in adult and pediatric patients to inform treatment strategies in the context of a pandemic. The tiered structure of this study allows for an adaptive approach as diagnostic, pathophysiologic, and therapeutic data pertaining to SARS-CoV-2 evolves. This study methodology may serve as a template to guide development of future consortia.

\footnotetext{
Author details

${ }^{1}$ Department of Neurology, NYU School of Medicine, New York, NY, USA. ${ }^{2}$ Division of Stroke and Neurocritical Care, Department of Neurology, The Ohio State University, Columbus, OH, USA. ${ }^{3}$ Division of Pediatric Critical Care Medicine, UPMC Children's Hospital of Pittsburgh, Pittsburgh, PA, USA. ${ }^{4}$ Departments of Anesthesiology and Critical Care Medicine, and Pediatrics, Johns Hopkins Children's Center, The Johns Hopkins University SOM, Baltimore, MD, USA. ${ }^{5}$ Primary Children's Hospital, University of Utah School of Medicine,
} 
Salt Lake City, UT, USA. ${ }^{6}$ Departments of Anesthesiology and Critical Care Medicine, Neurology, and Neurosurgery, The Johns Hopkins University School of Medicine, Baltimore, MD, USA. ${ }^{7}$ Division of Anaesthesia, Addenbrooke's Hospital, University of Cambridge, Cambridge, UK. ${ }^{8}$ Departments of Anesthesiology, Pediatrics, Bioengineering, and Clinical and Translational Science, Safar Center for Resuscitation Research, University of Pittsburgh, Pittsburgh, PA, USA. ${ }^{9}$ Neurocritical Care Unit, Department of Neurology, Medical University of Innsbruck, Innsbruck, Austria. ${ }^{10}$ College of Nursing, The Ohio State University, 760 Kinnear Rd, Columbus, OH 43212, USA. " ${ }^{11}$ Departments of Critical Care Medicine, Neurology, and Neurosurgery, Safar Center for Resuscitation Research, University of Pittsburgh School of Medicine, Pittsburgh, PA, USA.

\section{Acknowledgements}

The authors would like to thank the Adult Study Coordinator Team: Michal Hammond, MD; Valeria Altamirano, BS, MS; Carlos Villamizar-Rosales, MD; Ali Smith; Lauren E. Kaplan, BS; Nicole Paul, BS; Charith Ratnayake, BS; Aditya D. Sharma from the University of Pittsburgh and Haley Roberts, BS from The Ohio State University. We also thank the Pediatric Study Coordinator Team: Pamela Rubin, RN and David Maloney, BS from the Department of Critical Care Medicine, UPMC Children's Hospital of Pittsburgh, and Pediatric Neurointensivists: Dennis Simon, MD from the Department of Critical Care Medicine, UPMC Children's Hospital of Pittsburgh, Elizabeth Wells, MD, MHS from the Division of Neurology, Children's National Hospital, Washington, D.C., Juan Piantino MD Division of Neurology, The Oregon Health and Science University, and Andrew Geneslaw, MD, Department of Pediatrics, Morgan Stanley Children's Hospital. The authors gratefully acknowledge the support from the Neurocritical Care Research Central and The Neurocritical Care Research Network of the Neurocritical Care Society.

\section{Author contributions}

All authors have contributed equally to the work to meet criteria for authorship.

\section{Source of support}

This study is partially funded by a grant from the Clinical Translational Science Institute COVID 19 Pilot Award from the University of Pittsburgh.

\section{Conflicts of interest}

R21NS113037 (Chou), the University of Pittsburgh Dean's Faculty Advancement Award (Chou), Clinical and Translational Science Institute, University of Pittsburgh (Chou). Dr. Chou reports grant funding from the National Institutes of Health. Dr. Ziai reports grant funding from the National Institutes of Health, and personal fees from CR Bard and Portola outside the submitted work. Dr. Fink reports grant funding from The National Institute of Health. Dr. Helbok reports personal fees from BARD Medical, Zoll Medical Corporation, and Integra Lifesciences outside the submitted work. The remaining authors have nothing to disclose.

\section{Ethical approval/informed consent}

The study has been approved by The Institutional Review Board at The University of Pittsburgh, and by participating sites prior to data collection.

\section{Publisher's Note}

Springer Nature remains neutral with regard to jurisdictional claims in published maps and institutional affiliations.

\section{Published online: 22 May 2020}

\section{References}

1. Asadi-Pooya AA, Simani L. Central nervous system manifestations of COVID-19: a systematic review. J Neurol Sci. 2020;413:116832.

2. Poyiadji N, et al. COVID-19-associated acute hemorrhagic necrotizing encephalopathy: CT and MRI features. Radiology. 2020;1201187.

3. Li Y, Wang M, Zhou Y, Jiang C, Xian Y, Mao L, Hong C, Chen S, Wang Y, Wang H, Li M, Jin H, Hu B. Acute cerebrovascular disease following COVID-19: a single center, retrospective, observational study. The Lancet. 2020 (preprint).
4. Toscano G, et al. Guillain-Barre syndrome associated with SARS-CoV-2. N Engl J Med. 2020

5. Wu Y, et al. Nervous system involvement after infection with COVID-19 and other coronaviruses. Brain Behav Immun. 2020.

6. Mao L, et al. Neurologic manifestations of hospitalized patients with coronavirus disease 2019 in Wuhan, China. JAMA Neurol. 2020.

7. Klok FA, et al. Confirmation of the high cumulative incidence of thrombotic complications in critically ill ICU patients with COVID-19: an updated analysis. Thromb Res. 2020.

8. Zhao H, et al. Guillain-Barre syndrome associated with SARS-CoV-2 infection: causality or coincidence? Lancet Neurol. 2020;19(5):383-4.

9. Sedaghat Z, Karimi N. Guillain Barre syndrome associated with COVID-19 infection: a case report. J Clin Neurosci. 2020.

10. Camdessanche JP, et al. COVID-19 may induce Guillain-Barre syndrome. Rev Neurol (Paris). 2020.

11. Virani A, et al. Guillain-Barre syndrome associated with SARS-CoV-2 infection. IDCases. 2020; e00771.

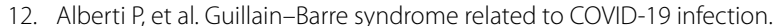
Neurol Neuroimmunol Neuroinflamm. 2020;7(4).

13. Padroni $M$, et al. Guillain-Barre syndrome following COVID-19: new infection, old complication? J Neurol. 2020.

14. Moriguchi T, et al. A first case of meningitis/encephalitis associated with SARS-coronavirus-2. Int J Infect Dis. 2020;94:55-8.

15. Helms J, et al. Neurologic features in severe SARS-CoV-2 infection. N Engl J Med. 2020.

16. Needham EJ, et al. Neurological implications of COVID-19 infections. Neurocrit Care. 2020

17. Hung EC, et al. Detection of SARS coronavirus RNA in the cerebrospinal fluid of a patient with severe acute respiratory syndrome. Clin Chem. 2003; 12:2108-9.

18. He $L$, et al. Expression of the monoclonal antibody against nucleocapsid antigen of SARS-associated coronavirus in autopsy tissues from SARS patients. Di Yi Jun Yi Da Xue Xue Bao. 2003;23(11):1128-30.

19. Hwang CS. Olfactory neuropathy in severe acute respiratory syndrome: report of A case. Acta Neurol Taiwan. 2006;15(1):26-8.

20. Yu F, et al. Measures for diagnosing and treating infections by a novel coronavirus responsible for a pneumonia outbreak originating in Wuhan. China. Microbes Infect. 2020;22(2):74-9.

21. $\mathrm{Xu}$ J, et al. Detection of severe acute respiratory syndrome coronavirus in the brain: potential role of the chemokine mig in pathogenesis. Clin Infect Dis. 2005;41(8):1089-96.

22. Ding $Y$, et al. Organ distribution of severe acute respiratory syndrome (SARS) associated coronavirus (SARS-CoV) in SARS patients: implications for pathogenesis and virus transmission pathways. J Pathol. 2004;203(2):622-30

23. Netland J, et al. Severe acute respiratory syndrome coronavirus infection causes neuronal death in the absence of encephalitis in mice transgenic for human ACE2. J Virol. 2008;82(15):7264-75.

24. Hoffmann M, et al. SARS-CoV-2 cell entry depends on ACE2 and TMPRSS2 and is blocked by a clinically proven protease inhibitor. Cell. 2020;181(2):271-280.e8.

25. Qi J, Zhou Y, Hua J, Zhang L, Bian J, Liu B, Zhao Z, Jin S. The scRNA-seq expression profiling of the receptor ACE2 and the cellular protease TMPRSS2 reveals human organs susceptible to COVID-19 infection. bioRxiv. 2020.

26. Paniz-Mondolfi A, et al. Central nervous system involvement by severe acute respiratory syndrome coronavirus-2 (SARS-CoV-2). J Med Virol. 2020.

27. Desforges $\mathrm{M}$, et al. Human coronaviruses and other respiratory viruses: underestimated opportunistic pathogens of the central nervous system? Viruses. 2019;12(1):14.

28. Machado C, Gutierrez J. Brainstem dysfunction in SARS-COV2 infection can be a potential cause of respiratory distress. 2020 (preprints).

29. Filatov A, et al. Neurological complications of coronavirus disease (COVID19): encephalopathy. Cureus. 2020;12(3):e7352.

30. Mlakar J, et al. Zika virus associated with microcephaly. N Engl J Med. 2016;374(10):951-8

31. Baltagi SA, et al. Neurological sequelae of 2009 influenza A (H1N1) in children: a case series observed during a pandemic. Pediatr Crit Care Med. 2010;11(2):179-84. 
32. Messacar K, et al. Acute flaccid myelitis: a clinical review of US cases 2012-2015. Ann Neurol. 2016;80(3):326-38.

33. Dong Y., et al. Epidemiology of COVID-19 among children in China. Pediatrics. 2020.

34. Aggarwal G, Lippi G, Michael Henry B. Cerebrovascular disease is associated with an increased disease severity in patients with coronavirus disease 2019 (COVID-19): a pooled analysis of published literature. Int J Stroke. 2020. 1747493020921664.

35. Nathan N, Prevost B, Corvol H. Atypical presentation of COVID-19 in young infants. Lancet. 2020.

36. Suarez II, Macdonald RL. The end of the tower of babel in subarachnoid hemorrhage: common data elements at last. Neurocrit Care. 2019;30(Suppl 1):1-3.

37. Suarez Jl, et al. Common data elements for unruptured intracranial aneurysms and subarachnoid hemorrhage clinical research: a national institute for neurological disorders and stroke and national library of medicine project. Neurocrit Care. 2019;30(Suppl 1):4-19.

38. Saver $\mathrm{L}$, et al. Standardizing the structure of stroke clinical and epidemiologic research data: the National Institute of Neurological Disorders and Stroke (NINDS) Stroke Common Data Element (CDE) project. Stroke. 2012;43(4):967-73.

39. Maas Al, et al. Common data elements for traumatic brain injury: recommendations from the interagency working group on demographics and clinical assessment. Arch Phys Med Rehabil. 2010;91(11):1641-9.

40. Chou SH, Macdonald RL, Keller E. Biospecimens and molecular and cellular biomarkers in aneurysmal subarachnoid hemorrhage studies: common data elements and standard reporting recommendations. Neurocrit Care. 2019;30(Suppl 1):46-59.
41. Hackenberg KAM, et al. Common data elements for radiological imaging of patients with subarachnoid hemorrhage: proposal of a multidisciplinary research group. Neurocrit Care. 2019;30(Suppl 1):60-78.

42. de Oliveira Manoel $\mathrm{AL}$, et al. Common data elements for unruptured intracranial aneurysms and aneurysmal subarachnoid hemorrhage: recommendations from the working group on hospital course and acute therapies-proposal of a multidisciplinary research group. Neurocrit Care. 2019;30(Suppl 1):36-45.

43. Damani $\mathrm{R}$, et al. Common data element for unruptured intracranial aneurysm and subarachnoid hemorrhage: recommendations from assessments and clinical examination workgroup/subcommittee. Neurocrit Care. 2019;30(Suppl 1):28-35.

44. Bijlenga $\mathrm{P}$, et al. Common data elements for subarachnoid hemorrhage and unruptured intracranial aneurysms: recommendations from the working group on subject characteristics. Neurocrit Care. 2019;30(Suppl 1):20-7.

45. Hill AB. The environment and disease: association or causation? 1965. J R Soc Med. 2015;108(1):32-7.

46. Mortimer PP. Was encephalitis lethargica a post-influenzal or some other phenomenon? Time to re-examine the problem. Epidemiol Infect. 2009;137(4):449-55

47. Vilensky J, Gilman S. Encephalitis lethargica: could this disease be recognised if the epidemic recurred? Pract Neurol. 2006;6:360-7.

48. McCall S, Vilensky J, Gilman S, Taubenberger J. The relationship between encephalitis lethargica and influenza: a critical analysis. J Neurovirol. 2009;14(3):177-85. 